\title{
Quality of Service and Performance Analysis with Reactive Protocol in Manet by a Fuzzy Scheduler
}

\author{
A.K. Daniel, Bharat Tripathi, Narendra Pal Singh \\ Department of Computer Science \& Engineering \\ Madan Mohan Malaviya Engineering College Gorakhpur(U.P)
}

\begin{abstract}
This paper presents an analysis of DSR protocol and packet scheduling algorithm to find those that most improve performance in congested network and proposes our algorithm to improve the performance of DSR protocol by using fuzzy logic in mobile ad hoc networks. Packet schedulers in wireless ad hoc networks serve data packets in FIFO order. Because, a scheduling algorithm to schedule the packet based on their priorities will improve the performance of the network. It is found that the scheduler provides overall improvement in the performance of the system when evaluated under different load and mobility conditions. The performance of this scheduler has been studied using NS2.34 simulator and measured such as packet delivery ratio, end-toend delay and throughput and an attempt to apply fuzzy logic in the design and implementation of a rule based scheduling algorithm to solve the shortcoming of well-known drop tail algorithms. Our main contribution is proposing a fuzzy approach to multi-packets scheduling in which the scheduling parameters are treated as fuzzy variables. It is concluded that the proposed fuzzy approach is very promising and it has the potential to be considered for future research.
\end{abstract}

\section{Keywords}

NS2.34, Fuzzy Priority Scheduler, DSR, MANETs

\section{INTRODUCTION}

The network which eliminates the need of physical infrastructure is usually termed as adhoc network. It is a collection of multiple hopes radio network and manages the connection in decentralized manner. In the current DSR there are many challenges like packet losses and new route discovery. So that due to this we faces the problem like packet delay and decrease throughput. To avoid this lacking we introduce a scheduler in MANET. A MANET is an autonomous group of mobile users that communicate over reasonably slow wireless links in an arbitrary manner.

The network topology may vary rapidly and unpredictably over time, because the nodes are mobile. The network is decentralized, where all network activity including discovering the topology and delivering messages must be executed by the nodes themselves. Hence routing functionality will have to be incorporated into the mobile nodes. MANET is a kind of wireless ad-hoc network and it is a self-configuring network of mobile routers (and associated hosts) connected by wireless links the union of which forms an arbitrary topology. Such a network may operate in a standalone fashion, or may be connected to the larger Internet [1].The mobile nodes can directly communicate to those nodes that are in radio range of each other, whereas others nodes need the help of intermediate nodes to route there packets.

The effects of scheduling algorithms on E-DSR are study by the NS2.34 simulator. In Fig. 1 nodes D and C must discover the route through $\mathrm{B}$ in order to communicate. are not in direct transmission range of each other [1].Since the mobile nodes in the network dynamically establish routing among themselves to form their own network is called infrastructure less network .All nodes of these networks behave not only as hosts but also as routers, forwarding packets to other mobile nodes in the network that may not be within direct wireless transmission of each other. The routing algorithms develop a route minimizing the time required to converge and bandwidth overhead at the same time enabling proper routing. Once the route is established, a scheduler schedules the packets on packet by packet basis. The simplest possible scheduling discipline is first in first out. The disadvantage of this technique is that it cannot differentiate among connections. Hence the choice of scheduling algorithm to determine which queued packet to process next will have a significant effect on overall end to end performance.

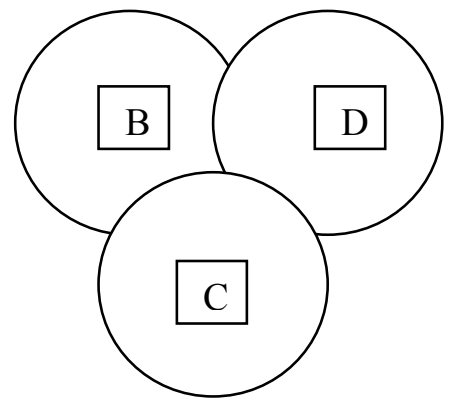

Figure 1: Mobile adhoc networks

In this paper we basically focuses on a fuzzy based priority scheduling scheme with expiry time concept, this improves the QoS parameters in wireless ad hoc network. Hence, introducing a scheduling algorithm to determine which queued packet to process within its time limit next will improve the overall end-to-end performance. Without scheduling, the packets will be processed in FIFO manner and hence there is frequently dropped the packets. A scheduler should schedule the packets to reach the destination quickly, which are at the verge of expiry. The fuzzy concepts were simulated using NS2 and the results of the fuzzy concepts were applied to the NS2.34. The performance of the proposed method has been evaluated. It has been observed that it 
improves the protocol in the aspects of delivery rate, control packets, dropped packets and end-to-end delay.

\section{DSR PROTOCOL DESCRIPTION}

In this section we describe the basic functionality of DSR and in the next sub-section the parameters for QoS have been discussed.

\subsection{DSR protocol}

Dynamic Source Routing (DSR) [2] is a reactive protocol it doesn't use periodic advertisements. It computes the routes when necessary and then maintains them. In DSR, when a node has a packet to send to some destination and does not currently have a route to that destination in its route cache, the node initiates Route discovery to find a route; this node is known as the initiator of the Route Discovery, and the destination of the packet is known as the Discovery's target. The initiator transmits a Route Request packet as a local broadcast, specifying the target and a unique identifier from the initiator. Each node receiving the Route Request, if it has recently seen this request identifier from the initiator, discards the Request. Otherwise, it appends its own node address to a list in the Request and rebroadcasts the Request. When the Route Request reaches its target node, the target sends a Route replay back to the initiator of the Request, including a copy of the accumulated list of addresses from the request. When the replay reaches the initiator of the request, it caches the new route in its route cache. The meaning of Routing maintenance refers to each DSR node maintains a route cache; it records the route information of node-by-node which can reach to the other nodes. Otherwise, every node can move from the data packet which is transmitting by the neighbor. The process of the snooping can be used to analysis the route information which is recorded in the front of data packet, the node records route information to its route cache if the route is a new one. Thus, more and more route information would be record to the route cache by the node and reduce the time of Flooding to broadcast RREQ. Meanwhile, the bandwidth of each node can also be saved. The processing of routing maintenance detects the changing of network topology, and it knows whether the route is still available or not.

Typically, as shown in Figure 1, [3] an intermediate node may not receive an acknowledgement from a neighboring node, triggering a Route Error packet to flow back to the source, informing Source and also all intermediate nodes of the link failure. In such a case all nodes, including source, will remove the route entry from their respective route cache. For example, Node 3 may not get an acknowledgement from Node 4, thus representing a route breakdown from $\mathrm{S}$ to $\mathrm{D}$. In such a case Node 3 will notice the failure first and it will inform the source about the link failure.

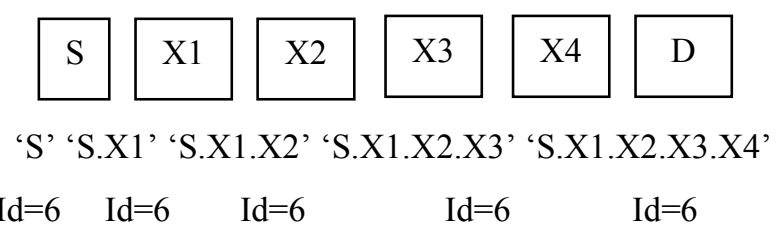

Figure 2: Route request packet flow in DSR

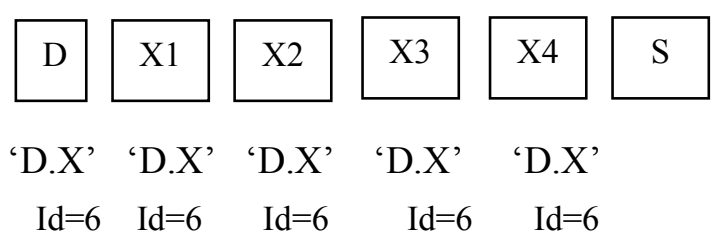

Figure 3: Route reply packet flow in DSR

\subsection{Process of packet scheduling in DSR}

In Dynamic Source Routing Protocol the packet is store in DSR cache in FIFO order. In DSR protocol the route of the packet is store in cache of every node. The packet is transfer from source to destination in FIFO order. The packet who comes first is transfer first and packet that comes last can transfer last. There are two types of packets in any complete information packets. Data packet and control packet, control packet is play vital role when transfer any information. The overall performance of packets is depending upon the control packet. So sometimes the data packets are transfer before the control packet due to first in first out scheduling. The problem accrues in the packet delivery ratio and packet sending performance is decrease. So we introduce the fuzzy scheduling concept, in packet queue, in this we used three variable describe in next chapter for setting the priority of the packet before it expired. Consider a routing path from the source node A to a destination node E as shown in Fig.4. The initial path is determined using the path discovery process, in which the distance between the source and destination is the shortest or very close to it. A packet takes three hops while following route from A to $\mathrm{E}$. In this new shape, B is in the transmission range of $\mathrm{A}$ and $\mathrm{C}$ is in the transmission range of B. Similarly D is in the transmission range of E. However, because of the usage of route caches and the validity of the existing routing information, the routing table entries are not updated. Using the routing paths shown in Fig.4, a packet still takes three hops to reach from $\mathrm{A}$ to $\mathrm{E}$ and needs only three hops by dynamically modifying the entries of the routing tables as in Fig.4.

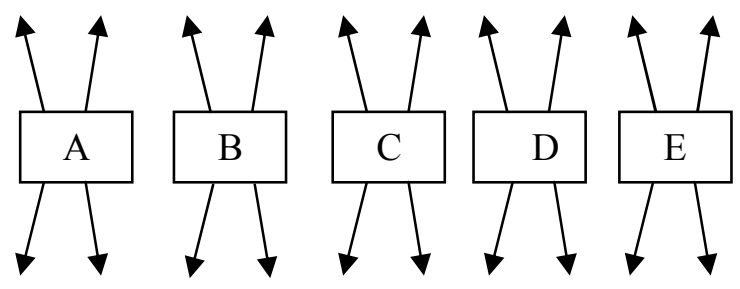

Figure 4: Different routing path of packet transfer using FIFO process 


\section{FUZZY SCHEDULAR IN DSR PROTOCOL}

In dynamic source routing, when a host needs a route to another host, it dynamically determines one based on cached information and on the results of a route discovery protocol. Due to the source routing characteristic [5] of DSR, overhead is increased. Moreover, during the route discovery process, each node takes part in forwarding Route Request (RREQ) packet $[7,8]$. Each node except the intended destination forwards the Route Reply (RREP) packet [7, 8] to create the route. Though, these RREPs increase the number of multiple paths to reach destination, they increase the control packet load of the network. Our proposal is to modify the basic DSR to change the packet scheduling (drop tail) into fuzzy scheduling.

Table 1: Scheduling of packets in different nodes in DSR protocol using FIFO

\begin{tabular}{|c|c|c|c|c|}
\hline A & B & C & D & E \\
\hline P1 & P1 & P1 & P1 & P1 \\
\hline P2 & P2 & P2 & P2 & P2 \\
\hline P3 & P3 & P3 & P3 & P3 \\
\hline
\end{tabular}

\subsection{Fuzzy algorithm}

There are two basic approaches or algorithm used in fuzzy logic. The first one is Mamdani and second is Sugeno both the algorithm developed for fuzzy concept. In this paper we use the Mamdani algorithm for developing the concept of fuzzy scheduler in dynamic source routing protocol. The description of Mamdani algorithm is given below.

Loop \| System is running for ever

For each DSR packet queue the queue

Do the following

Step 1: for each ready packet $P$ (a packet which is keep in packet queue and ready to transmite)

Feed it into the rule base schedule System engine.

Consider the output of fuzzy system is priority of packet $P$.

Step 2: execute the packet with higest priority until any scheduling event accure (a running packet is finishes, until a new packet is arrives)

Step 3: update the DSR queue END

END loop $\|$

\subsection{Fuzzy rule based scheduler}

A modified rule-based fuzzy scheduler that deals with both task priority and its execution time is presented in this section. A fuzzy-based decision maker (FDM) has a modified rulebased fuzzy scheduler that deals with both task priority and its execution time is presented in this section. A fuzzy-based decision maker (FDM) has been proposed to compute the new priority (Pn) of all packets according to the packets priority (Po) and its expiry time (Ex), as shown in table 2. The measured variables are inverted into suitable linguistic variables. In this application, the following linguistic variables are used for priority $(\mathrm{Po})$, and new calculated priority $(\mathrm{Pn})$; Very Low (VL), Low (L), Medium (M), High (H), and Very High (VH). The fuzzy sets definition for expiry time (Ex) are Short (S), Medium (M), High (H), Figure (5) shows the universe of discourse and fuzzy sets of these variables. Fuzzy sets can have a variety of shapes. However, a triangular or a trapezoid can often provide an adequate representation of the knowledge [11]. The proposed fuzzy decision maker is a collection of linguistic rules which describe the relationships between measured variables (Po \& Ex), and calculated output $(\mathrm{Pn})$. Table 2 contains rules, since we have three fuzzy sets for each variable. Each rule is represented by IF and THEN statement such as; IF MPx and LEx THEN VLPn this means that if the priority is very low (VLPo) and the Ex time is short (STx), then the new calculated priority is high (HPn).

Table 2: Fuzzy rule base

\begin{tabular}{|c|c|c|c|}
\hline Hopx & L & M & P \\
\hline Px & \multicolumn{4}{|c|}{ Expiry time(Low) } \\
\hline \multicolumn{4}{|c|}{} \\
\hline L & L & L & VL \\
\hline M & VL & VL & VL \\
\hline H & L & VL & VL \\
\hline \multicolumn{5}{|c|}{ Expiry time(Medium) } \\
\hline L & M & M & L \\
\hline M & M & M & L \\
\hline H & M & M & M \\
\hline \multicolumn{5}{|c|}{ Expiry time(High) } \\
\hline L & VH & VH & H \\
\hline M & H & M & M \\
\hline H & H & H & M \\
\hline
\end{tabular}

The Mamdani-style inference process is used [11], and the center of gravity defuzzification method is applied to convert the fuzzy output into a crisp value that represents the new priority of a task. It is clear that the average waiting time and average turnaround time obtained from the FuzS algorithm are better than that obtained from the FifoS algorithm and close to that obtained from the SFJ algorithm. T Mamdani's fuzzy inference method proposed in 1975 by Ebrahim Mamdani [8] in this method we take the fuzzy inputs and procedure of fuzzifying is process with fuzzy operator. There are also another fuzzy method called Sugeno.The main difference between Mamdani and Sugeno is that the Sugeno output membership functions are either linear or constant but Mamdani's inference expects the output membership functions to be fuzzy sets.

\subsection{Determine fuzzy sets}

Fuzzy sets can have a variety of shapes. However, a triangle or a trapezoid can often provide an adequate representation of the expert knowledge, and at the same time, significantly simplifies the process of computation. The quality of fuzzy approximation depends on the quality of the rules. The result always approximates some unknown non linear function that can change in time. Fuzzy systems theory or "fuzzy logic" is a linguistic theory that models how we reason with vague rules of thumb and commonsense. The basic unit of fuzzy function approximation is "if then" rules. A fuzzy system is a set of if then rules that maps input to output. 
The result of learning algorithm is a family of fuzzy relation defined on the product space $\mathrm{Z}$.For each fuzzy relation $\mathrm{R}^{(\mathrm{r})}$ $=\mathrm{A} 1^{(\mathrm{r})} \ldots \ldots . \mathrm{An}_{(\mathrm{r})} \mathrm{X} \mathrm{B1}^{(\mathrm{r})} \mathrm{XXX} \mathrm{Bm}_{(\mathrm{r})}$ a fuzzy rule is straight forwardly derived based on the following schema.

IF $\mathrm{X} 1$ is $\mathrm{A} 1{ }^{(\mathrm{r})} \mathrm{AND} \ldots \ldots$ AND $\mathrm{X}_{\mathrm{n}}$ is $\mathrm{An}^{(\mathrm{r})}$

THEN $\mathrm{Y} 1$ is $\mathrm{B} 1^{(\mathrm{r})}$ AND ......AND $\mathrm{Y}_{\mathrm{m}}$ is $\mathrm{Bm}{ }^{(\mathrm{r})}$

Table 3: Scheduling of packets in different nodes in DSR protocol using FS

\begin{tabular}{|c|c|c|c|c|}
\hline A & B & C & D & E \\
\hline P3 & P3 & P3 & P3 & P3 \\
\hline P1 & P1 & P1 & P1 & P1 \\
\hline P2 & P2 & P2 & P2 & P2 \\
\hline
\end{tabular}

\section{PERFORMANCE EVALUATION}

The simulation for evaluating the fuzzy scheduler was implemented within the NS2.34 library. The simulation package NS2.34 is used to analyze and evaluate the performance of the proposed fuzzy scheduler. The NS2.34 [8][9], software that provides scalable simulations of wireless networks. In this simulation, we consider a network of 5, 10, 25, 50, 100 nodes (one source and one destination) placed in A dimension of $300 \mathrm{~m} \times 300 \mathrm{~m}$ area. Each simulation is run for 800 seconds of simulation time. Multiple simulations run with different seed values were conducted for each scenario and collected data was averaged over those runs. Table 4 lists the simulation parameter.

Table 4: Simulation parameter

\begin{tabular}{|c|c|}
\hline \multicolumn{2}{|c|}{ Simulation Parameter } \\
\hline Area & $300 \mathrm{~m} \times 300 \mathrm{~m}$ \\
\hline Simulation Time & $800 \mathrm{sec}$ \\
\hline No. of Nodes & $6,10,25,50,100$ \\
\hline Node Position & Random \\
\hline Mobility & Random Way Point \\
\hline Stop Time & $800 \mathrm{sec}$ \\
\hline Start Time & $10 \mathrm{sec}$ \\
\hline Max. Speed & $20 \mathrm{mps}$ \\
\hline Traffic Agent & CBR, TCP \\
\hline Packet Length & 512 Byte \\
\hline MAC Layer & 802.11 \\
\hline
\end{tabular}

4.1. Packet delivery ratio: Packet delivery ratio is the ratio of the number of data packets actually delivered to the destinations to the number of data packets supposed to be received.This number presents the effectiveness of the protocol.

4.2. Average end-to-end delay: End-to-end delay indicates how long it took for a packet to travel from the source to the application layer of the destination.

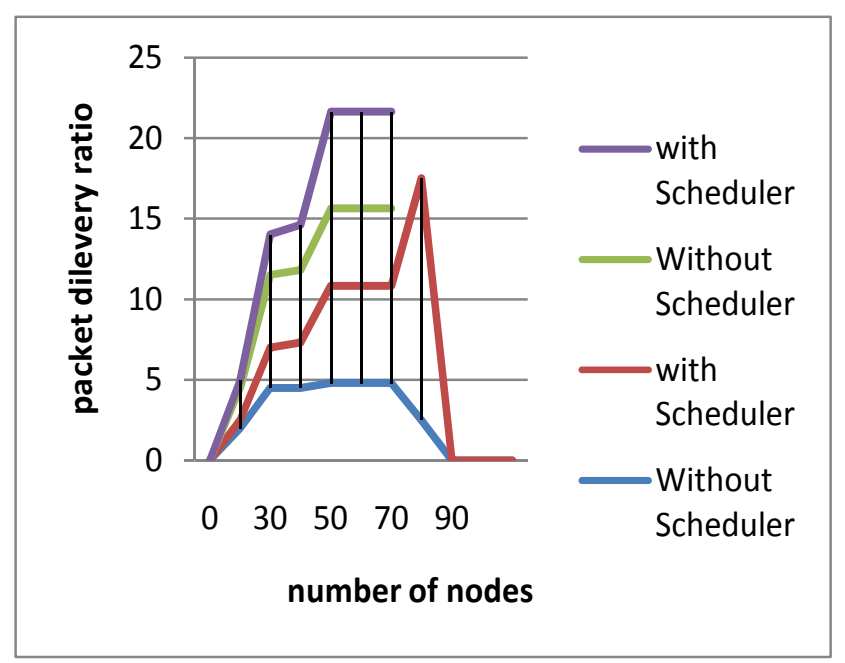

Figure 5: Average delivery ratio

4.3. Throughput: This is measured in bytes per sec, which also serve as the performance measure for the scheduler. The effects of setting priority to data packets, varying the number of sender's .The delay curve of DSR and EDSR protocols is shown in Figure 6. After inclusion of FPS, the delay performance is again evaluated and plotted.

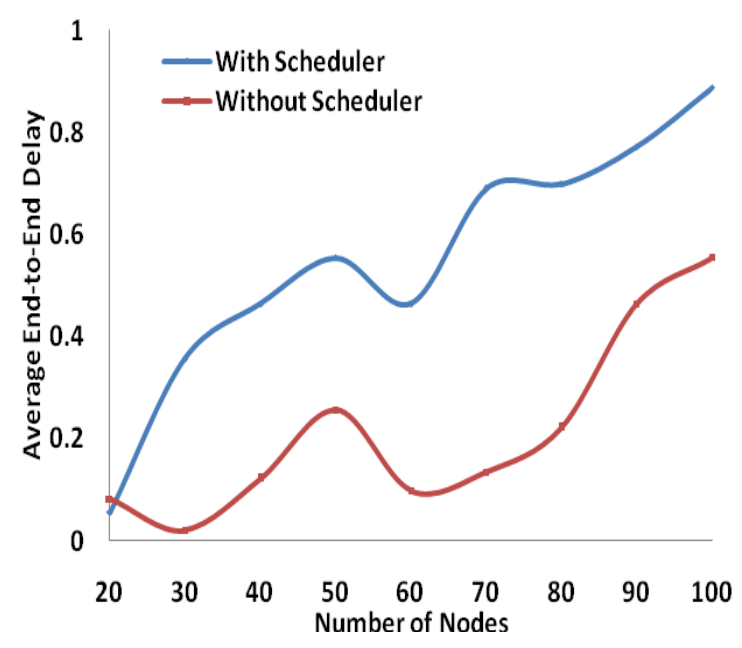

Figure 6: Average end-to end delay

\section{CONCLUSION}

We have addressed a fuzzy based priority scheduler for data packets, which improves the quality of service parameters in mobile ad hoc networks. The fuzzy scheduler attaches a priority index to each packet in the queue of the node. Unlike the normal sorting procedure for scheduling packet, a crisp priority index is calculated based on the inputs such as queue length, data rate, and expiry time of packets, which are derived from the network. The membership functions and rule bases of the fuzzy scheduler are carefully designed. The coding is done in TCL language and the output is verified using network simulator 2.34 fuzzy logic toolbox with VIM editor. From the simulation results, we find that giving high priority to control traffic should be carefully evaluated for use depending on the routing protocol. We show that on-demand 
routing protocols are likely to benefit from this arrangement, but proactive routing protocols might not. With scheduling algorithms using short distance metrics, data packets can be delivered much faster in a congested network, without additional control packet exchange for the algorithms. Furthermore, the implementation of these algorithms is simple. Thus, they are easily deployable to improve performance in resource-constrained ad hoc networks.

\section{REFERENCES}

[1] David B. Johnson, David A. Maltz, Yih-Chun Hu, and Jorjeta G. Jetcheva. February 2002 "The Dynamic Source Routing Protocol for Mobile Ad Hoc Networks". IETF Internet draft, Mobile Ad-hoc Network Working Group, IETF, (work in progress).

[2] Charles E. Perkins, Elizabeth M. Royer, and Samir Das. January 2002 "Ad Hoc On Demand Distance Vector (AODV) Routing". IETF Internet draft, Mobile Adhoc Network Working Group, IETF.

[3] J. Broch, D.A. Maltz, D.B. Johnson, Y.C. Hu, and J. Jetcheva. , October 1998, "A performance comparison of multi-hop wireless ad hoc network routing protocols". In Proceedings of ACM/IEEE MOBICOM, Dallas, TX.

[4] Samir R. Das, Charles E. Perkins, and Elizabeth M. Royer, March 2000 "Performance comparison of two ondemand routing protocols for ad hoc networks". In Proceedings of the IEEE INFOCOM, Tel- Aviv, Israel.

[5] P. Johansson, T. Larsson, N. Hedman, B. Mielczarek, and M. Degermark., August 1999,"Scenario-based
Performance Analysis of Routing Protocols for Mobile Ad-hoc Networks". In Proceedings of ACM MOBICOM, Seattle, WA.

[6] V.D. Park and M.S. Corson. April 1997, “A Highly Adaptive Distributed Routing Algorithm for Mobile Wireless Networks". In Proceedings of IEEE INFOCOM, Kobe, Japan.

[7] Bart Kosko, "Fuzzy Engineering", Prentice Hall International Inc. Second Edition 1998.

[8] The Network Simulator - ns2.http://www.isi.edu/nsnam/ns.

[9] C.Gomathy and S.Shanmugavel, "Implementation of modified Fuzzy Priority Schedule for MANET and performance analysis with mixed traffic, "in Proc.11thNationalConference.

[10] C.Gomathy and S.Shanmugavel "Fuzzy based Priority Scheduler for mobile adhoc networks" in Press.

[11] J. A. Freebersyser and B. Leinerr, 2001, "A DoD perspective on mobile ad hoc networks," in Ad Hoc Networking, C. E. Perkin, Ed. AddisonWesley, pp. 29-51.

[12] B. Leiner, R. Ruth, and A. R. Sastry, December, 1999. "Goals and challenges of the DARPA GloMo program," IEEE Personal Communications, vol. 3, no. 6, pp.

34-43, 